\title{
Myofascial Pain Dysfunction Syndrome Treatment by Occlusal Equilibration Using T-Scan and Electromyography for the Precise Analysis of Muscle Activity
}

\author{
Prafulla Thumati ${ }^{1}$, S. Padmaja ${ }^{2}$ \\ ${ }^{1}$ Department of Prosthodontics, Dayananda Sagar College of Dental Sciences, Bengaluru, Karnataka, India, ${ }^{2}$ Department of \\ Prosthodontics, Bapuji Dental College and Hospital, Davangere, Karnataka, India
}

Email for correspondence: drpadma28@gmail.com

\begin{abstract}
Context: A relationship between functional disorders of the masticatory system and headache was reported already in 1956, with spectacular reduction in chronic/recurrent headaches by the use of biteplanes. There are many types of headaches and many etiologic possibilities and it is extremely important that a differential diagnosis is made before. One of them is myofascial pain dysfunction syndrome which requires such diagnosis and treatment. Aims and Objectives: To report the reports the results of patients with chronic myofascial pain syndrome (MFPDS) treated by occlusal equilibration using immediate complete anterior guidance development (ICAGD) by Kerstein as the treatment option. Materials and Methods: Seventy five patients diagnosed as myofascial pain dysfunction syndrome cases were taken up to be treated by ICAGD technique as described by Kerstein. The common complaints of the patients were pain in the masseter \& temporal muscles, jaw tiredness in the mornings, night bruxing \& difficulty in chewing. This technique involves occlusal equilibration to remove all posterior interferences and establish anterior guidance. The patients were treated over 3 visits one week apart and followed for three years. Results: The symptoms reduction occurred for all the patients after the first correction in about 5-10 days. In about a period of 3 years review, no recurrence was seen of the chronic myofascial symptoms. Conclusion: In the present study it is seen that the treatment of 75 patients with MFPDS with occlusal equilibration using digital analysis of occlusion by T-scan, electromyography and joint vibration analysis technology which is quantifying of occlusal forces against time has brought relief of symptoms in about week to ten days time.
\end{abstract}

Key words: Electromyography, enameloplasty, immediate complete anterior guidance development, joint vibration analysis, myofascial pain syndrome, occlusal discrepancy, T-scan

\section{INTRODUCTION}

A relationship between functional disorders of the masticatory system and headache was reported already in 1956, with a spectacular reduction in

\section{Quick Response Code Article Info:}

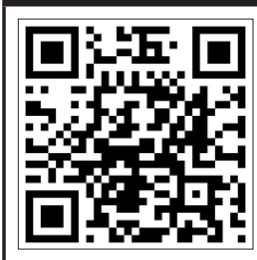

\begin{tabular}{l} 
doi: 10.5866/2017.9.10134 \\
\hline Received: $23-07-2017$ \\
Revised: $30-08-2017$ \\
Accepted: $15-09-2017$ \\
Available Online: $27-11-2017$ (www.nacd. \\
in)@ NAD, 2017 - All rights reserved
\end{tabular}

chronic/recurrent headaches by the use of bite planes. ${ }^{[1,2]}$ There are many types of headaches and many etiologic possibilities, and it is extremely important that a differential diagnosis is made before.

Occlusion is one of the fields of dentistry in which much overtreatment and in some cases faulty treatment have been performed. When we speak about the need for treatment, all kinds of treatment should be considered, from counselling, behavioral therapy, physiotherapy, and rest of various kinds of occlusal therapy. The question is always if anything of benefit can be offered to the patient that will 
provide relief of his/her signs and symptoms. Thus, any patient with soreness of jaw muscles, limitation of jaw movement, or impairments of temporomandibular joint (TMJ) function could be considered as candidates for occlusal therapy.

The chronic myofascial pain and dysfunction syndrome (MFPDS) signs and symptoms are pain in the face, limited mouth opening, muscle tenderness, deviation of the jaw on opening, noises in the TMJ, and pain in and around TMJ area. ${ }^{[3]}$ The etiologic factors and treatment modalities for chronic MFPDS are widely studied and debated.

Occlusal therapy constitutes a deliberate intervention for the purpose of changing the occlusal status. It should be based on a specific diagnosis and evaluation in each case. It has been reported that the reduced dysfunctional activity with the use of bite planes would return if the bite planes were left out of the mouth at night; however, it did not return for patients who had occlusal adjustment, even if they stopped using the bite planes. ${ }^{[4-6]}$

Occlusal equilibration as a treatment modality for relieving the symptoms of MFPDS has been successful for some people and not so for some people. ${ }^{[7]}$ This study reports the results of 75 patients of chronic MFPDS treated by occlusal equilibration using immediate complete anterior guidance development (ICAGD) by Kerstein as the treatment option. ${ }^{[8]}$ The present study reports the result of 75 patients with chronic MFPDS treated by "occlusal equilibration using ICAGD technique" given by Kerstein as the treatment option with Computer-guided ICAGD enameloplasty and simultaneously recorded T-scan, electromyography (EMG), and joint vibration analysis (JVA).

\section{MATERIALS AND METHODS}

The present study included 75 patients diagnosed as chronic MFPDS cases with 22 men and 53 women, having an age range of 18-60 years. Of this, 11 men were between 18 and 35 years and 11 of them between 36 and 60 years. 41 women fell in the age group of 18 and 35 years and 12 of them between 36 and 60 years. The areas of pain reported are in the region of masseter, temporalis, and lower border of the mandible near the molar area and in the ear or TMJ region [Table 1].

All the patients were screened with the diagnostic investigations such as orthopantomogram, diagnostic models, and biometric investigations such as T-scan, EMG, and JVA to study existing dentition. Using T-scan and EMG, the tooth contact sequence, changing occlusal forces, and fluctuating muscle activity levels of the temporalis and masseter muscle were recorded from an intercuspation followed by excursive movements till the muscle shutdown occurred.

About the location and nature of pain, a total of 147 sites of chronic discomfort with some patients showing more than one area were recorded. In this, temporalis area showed the maximum at 67 times and then the masseter area 57 times. The onset of the pain or discomfort was described by 67 people as tension and pain in the temporal area, and the next pattern was pain in the masseter area while chewing and stiffness in the face in the mornings. Thirty-one patients had unilateral or bilateral joint disturbances in the form of clicks or pops.

The symptom distribution subjectively has been summarized in Table 2.

\section{Technique of ICAGD treatment protocol}

The protocol for ICAGD is to start by recording the traces of all mandibular movements using T-scan [Figures 1-3]. Once the traces are studied for occluding time and discluding time details, the occlusal interferences in excursive mandibular movements are eliminated such as all Class I to III interferences described by Glickman. Once this is achieved, either by reduction enameloplasty or by addition to teeth, immediate posterior disclusion

\begin{tabular}{ll} 
Table 1: Regions of chronic pain & \\
$\begin{array}{l}\text { Regions of chronic } \\
\text { pain }\end{array}$ & N (\%) \\
\hline Masseter/buccinator & $57(76)$ \\
Temporalis & $67(89)$ \\
Lower border of mandible & $14(18.5)$ \\
TMJ region & $9(12)$ \\
\hline
\end{tabular}

TMJ: Temporomandibular joint

\section{Table 2: Subjective distribution of symptoms}

Subjective symptom distribution

Temporal headaches

Jaw pain in the masseter area

Jaw fatigue on chewing

TMJ area pain and noises

Night bruxing and night guards

TMJ: Temporomandibular joint 


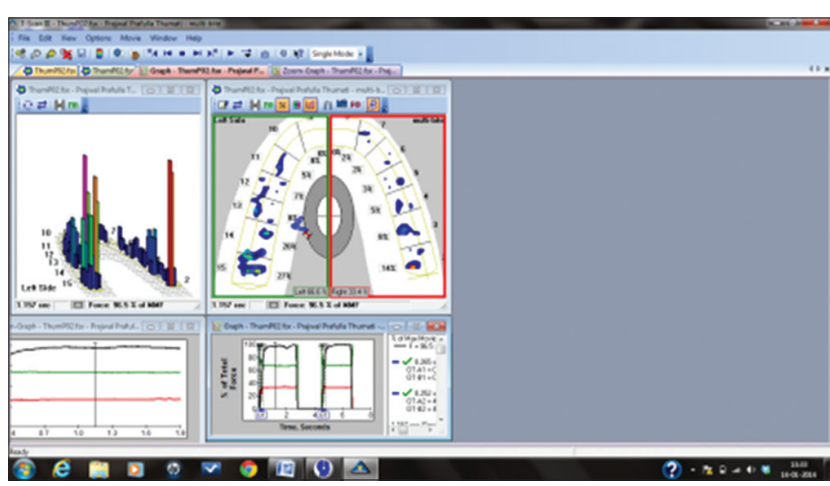

Figure 1: Pre-operative records (multi-bite)

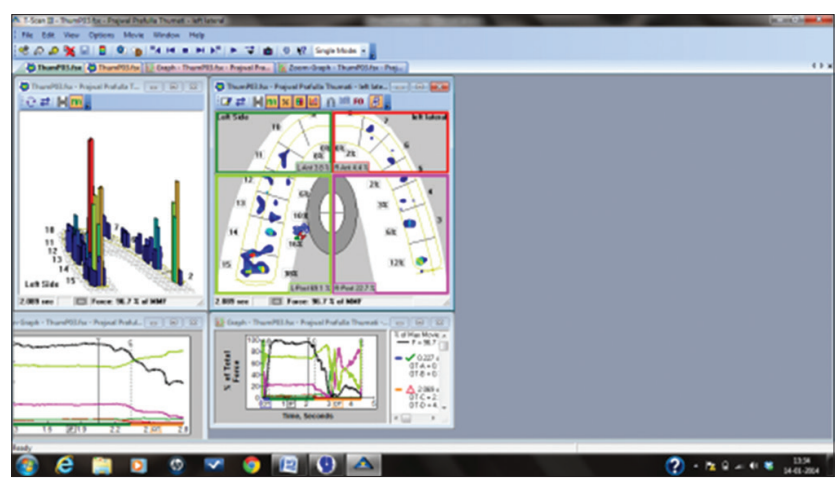

Figure 2: Left lateral recording

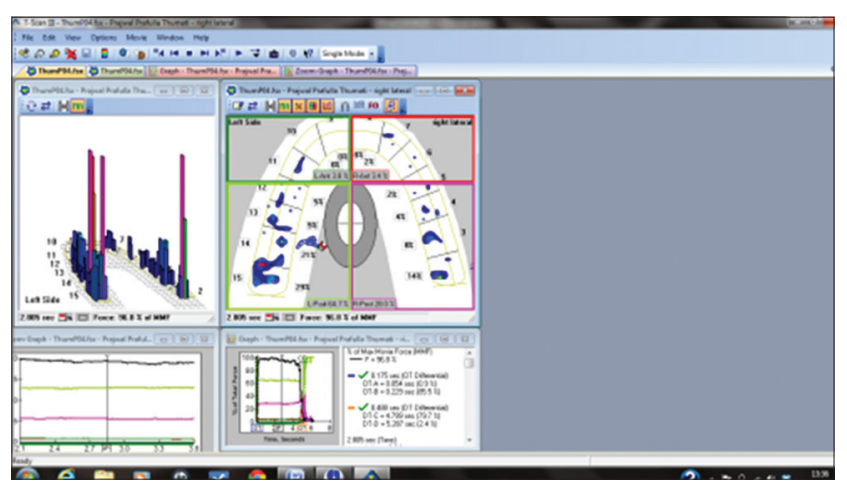

Figure 3: Right lateral recording

in $<0.5 \mathrm{~s}$ should be achieved. The intentions are to establish ICAGD with $<0.5 \mathrm{~s}$ disclusion time in excursive mandibular movements.

When we equilibrate occlusion by ICAGD technique, keep asking the patient - where is the highest pressure point in the bite, do you have any rocking points, is it equal on the left and right side, is it very light at the back end when you bite, is it hurting in temples, face, neck,or ears when you bite, do you feel blocked when you want to glide from side to side, is there raised tension in your face when you bite, and do you have anything that you do not like about your bite.

ICAGD is done in 2 phases. In the first phase, after analyzing the digital data of T-scan, enameloplasty (reduction) or addition to teeth with composites is done in such a way, and immediate posterior disclusion in $<0.5 \mathrm{~s}$ is achieved. This reduces muscular contraction in masticatory muscles by suppressing the mechanoreceptor activity due to shortened disclusion time. Thereby, lessened lactic acid produced with the increased availability of oxygen for the muscular tissues. This reduced muscle hyperactivity will be immediately shown as muscle relaxation, decreased pain, and stress released from the face for the patients'. Posttreatment records are taken again [Figures 4-6].

In the second phase, on $8^{\text {th }}$ day (after 1 week), pre- and post-treatment records were recorded. Occlusal interferences are looked for and cleared till the new habitual bite is eventless by having $<0.5 \mathrm{~s}$ disclusion time and the entire craniofacial physiology is at its best health. This is confirmed with the Bio-metric reports of T-scan, EMG, and JVA and disappearance of MFPDS symptoms over a period of 4-6 weeks completely. The patient was reviewed periodically to avoid changes in disclusion timings at regular interval of 1-, 3-, 6-months and 1 year. Follow-up visits showed that, after reduction, the pre-treatment levels of excursive muscular hyperactivity were lessened. ${ }^{[9]}$

The arch relation between maxillary and mandibular teeth is as follows: 59 of them Angles Class 1, 7 of them Class 2 inclusive of both the divisions, and 9 of them Class 3 . All of them were subjected to complete occlusal examination, which revealed that all of them had centric relation to centric occlusal (CR-CO) discrepancy of 0.25 $3 \mathrm{~mm}$ both in horizontal and vertical directions. In this, 49 patients had gone through orthodontics corrections. Even though they had good vertical relationship, 11 people had posterior crossbites and 4 of them anterior open bite.

All these patients were having an inability or limited ability to right and/or left excursive movements when anterior teeth are in contact. This was primarily due to both working and non-working interferences in the posterior teeth [Figures 1 and 2]. Some of these patients could move a bit from their habitual maximum contact, and others move laterally if they first separated their teeth vertically. Twentyone of them had protrusive interferences too, even 


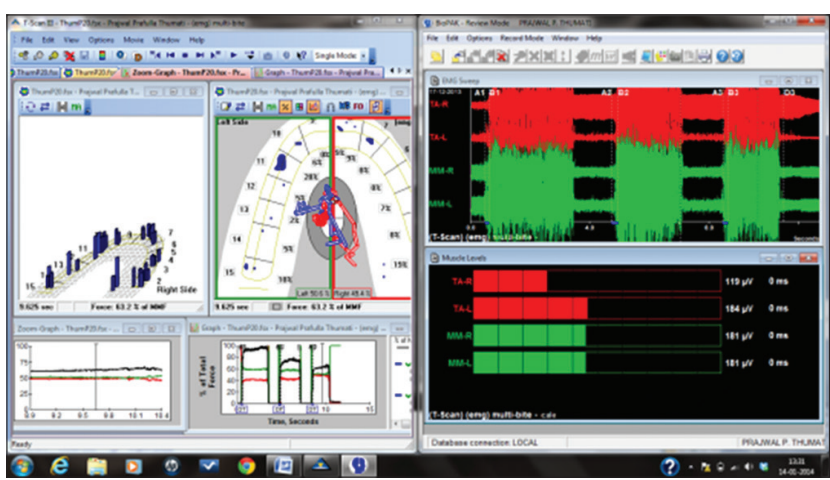

Figure 4: Post-treatment recording (multi-bite)

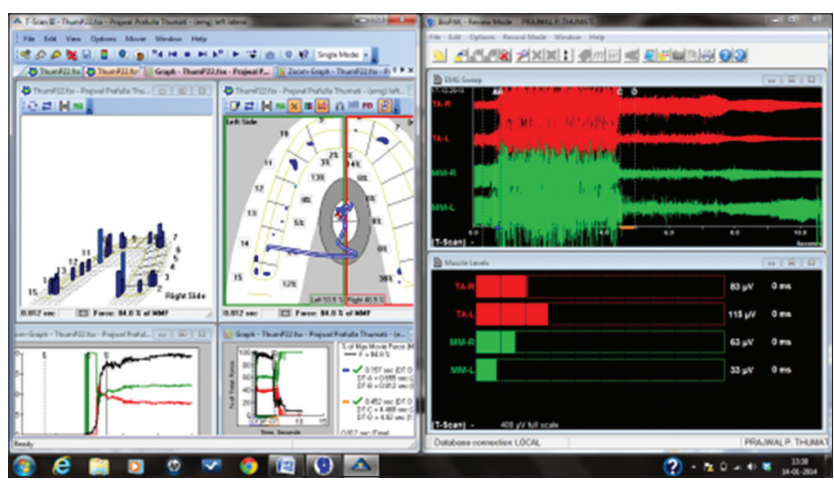

Figure 5: Post-treatment left lateral

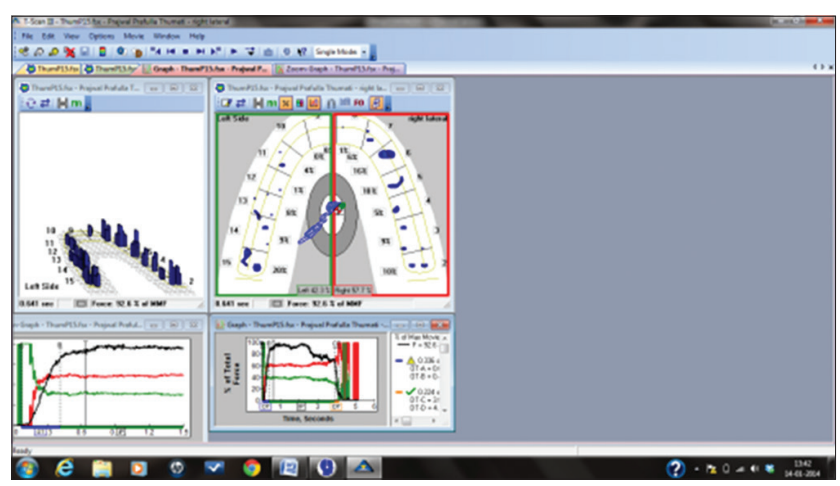

Figure 6: Post-treatment right lateral

though all of them could protrude mandible. Most of them had anterior worn out (wear out facets) incisal edges, especially canines, thereby anterior guidance being lost [Figure 3]. The 23 patients who were using acrylic resin splints (night guards) were subjected to occlusal evaluation without the splints. All these patients were unable to do lateral movements from ICP due to working and non-working interferences. The major factor in all these patients was missing freedom for the mandibular excursive movements in all directions in contact with the opposing teeth due to lack of anterior guidance. Since this is a subjective description of the findings, no control group is used.

\section{RESULTS}

Treatment objective was to free the contact mandibular movements in all eccentric directions by occlusal equilibration as described by Glickma and Kersteins ICAGD techniqu. However, no attempt is being made to modify the closure position to CR and neither an attempt was made to correct the CR-CO discrepancy. ${ }^{[10]}$ The sole treatment purpose was to remove all non-working and working interferences and develop an anterior guidance. For patients with anterior open bite and Class II angles relation, guidance was established on canines and first premolars, Class III angles relation, it was provided on incisors and canines, and Class I relation, it was on canines and incisors.

After the first visit for occlusal equilibration, all patients in the second visit after 1 week reported of easy jaw movements and fresh feeling in the face and lack of stiffness in the face indicating stress-free facial tissues and no muscle pain. Sixty-seven patients were very happy, five of them still complained of 10-20\% of symptoms who had few more interference. After the second visit corrections, even these five patients were very much comfortable. The other three patients were having flat occlusal surfaces and needed a couple of visits to gain anterior guidance after which even they were comfortable.

Regardless of the chronic nature of the patient's symptoms, symptom reduction occurred in a week's time. This agrees with the studies of Kerstein and others. All the 75 patients reported no facial tension or fatigue on eating. The bruxing patients reported of relaxed muscles and no pain. The episodes of bruxing had reduced as reported by their spouses or relatives. In the patients who complained of pain near ear or TMJ, the frequency of pain greatly reduced but the clicking and popping persisted. These patients recalled over 3 years showed that the results were maintained and no recurrence was seen.

\section{DISCUSSION}

From the data, it is seen that these problems are predominantly seen in women aged between 18 and 36 years; as 53 patients are women, out of 75 and 52 patients are between 18 and 35 years of age out of 75 . 
As per Glickman's classification, the maximum number of interferences were Class III, followed by Class II and Class I. ${ }^{[11]}$ Removal of class III interferences on non-working side during equilibration procedure, which is on the buccal inclines of the upper palatal cusps and lingual inclines of the lower buccal cusps, will facilitate better visualization of Class II and I interferences on the working side. Once, all the faulty contacts are eliminated and anterior guidance established with all freedom of Jaw movements.

As reported by Lundquist and Williamson, Dawson, and Kerstein, there will be masticatory muscle activity leading to a contraction in the muscles during closed position, and this will continue when the posterior teeth are in contact during excursive movement. ${ }^{[12]}$ In turn, this will lead to the release of contractile by-products such as lactic acid which results in muscle spasm, muscle fatigue, and pain. Due to spasmodic contractions, blood supply is reduced with decreased muscle function, oxygen, and increased ischemic changes. ${ }^{[13]}$ This will lead to increased pain as nerve ending are sensitive to lactic acid and ischemic changes.

Since the non-working and working interference were removed and anterior guidance was established, the excessive muscle activity is being interrupted during closing contacts giving rest to muscles. This will eliminate the muscle hyperactivity, fatigueness, tenderness, and pain in the muscles relieving the symptoms of all MFPDS symptoms from musculoskeletally based issues.

In all the 75 patients, $49(65 \%)$ of them have developed symptoms long after their orthodontic treatment. This gives a direction to think about, mere establishment of ideal vertical tooth relation may not lead to functionally and neuromuscularly healthy excursive movements. If free excursive mandibular movements are not established after, post-orthodontic treatment may lead to muscle dysfunction at later years. Thus, equilibration should be done to establish good and free functional movements without any interference after orthodontic treatment is completed and the teeth have assumed stable positions.

\section{CONCLUSION}

In the present report, it is seen that the treatment of 75 patients with MFPDS with occlusal equilibration using digital analysis of occlusion by T-scan, EMG, and JVA technology which is quantifying of occlusal forces against time has brought relief of symptoms in about week to 10 day time. Further, long-term and control studies are required to know the connection between neuromuscular health and teeth contacts.

\section{REFERENCES}

1. Berlin R, Dessner L. Bruxism and chronic headache. Lancet 1960;2:289-91.

2. Berlin R, Dessner L, Aberg S. Chronic headache and dysfunction of the temporo-mandibular joint. Acta Med Scand 1956;154:167-76.

3. Greene CS, Laskin DM. Long-term evaluation of conservative treatment for myofascial pain-dysfunction syndrome. J Am Dent Assoc 1974;89:1365-8.

4. Lederman $\mathrm{KH}$, Clayton JA. Patients with restored occlusions. Part III: The effect of occlusal splint therapy and occlusal adjustments on TMJ dysfunction. J Prosthet Dent 1983;50:95-100.

5. Solberg WK, Clark GT, Rugh JD. Nocturnal electromyographic evaluation of bruxism patients undergoing short term splint therapy. J Oral Rehabil 1975;2:215-23.

6. Solberg WK. Myofascial pain and dysfunction. In: Clark J, editor. Clinical Dentistry. Vol. 2. Ch. 37. Hagerstown, MD Harper \& Row; 1976.

7. Butler JH, Folke LE, Bandt CL. A descriptive survey of signs and symptoms associated with the myofascial paindysfunction syndrome. J Am Dent Assoc 1975;90:635-9.

8. Robert BK, Farrell S. Treatment of myofascial paindysfunction syndrome with occlusal equilibration. J Prosthet Dent 1990;63:695-700.

9. Riise C, Sheikholeslam A. The influence of experimental interfering occlusal contacts on the postural activity of the anterior temporal and masseter muscles in young adults. J Oral Rehabil 1982;9:419-25.

10. Helsing G. Occlusal adjustment and occlusal stability. J Prosthet Dent 1978;40:438-41.

11. Glickman I. Clinical Periodontology. $5^{\text {th }}$ ed. Philadelphia, PA: WB Saunders Co.; 1979. p. 953.

12. Williamson EH, Lundquist DO. Anterior guidance: Its effect on electromyographic activity of the temporal and masseter muscles. J Prosthet Dent 1983;49:816-23.

13. Dawson PE. Evaluation, Diagnosis and Treatment of Occlusal Problems. $2^{\text {nd }}$ ed. St Louis: CV Mosby Co.; 1988. p. 96. 